\title{
Bazedoxifene activates the angiotensin II-induced HUVEC hypertension model by targeting SIRT1
}

\author{
QIAN YU ${ }^{1}$, JIN ZHAO ${ }^{2}$ and BAOTANG LIU ${ }^{2}$ \\ ${ }^{1}$ Department of Cardiovascular Medicine, Sichuan Chengdu Fifth People's Hospital, Chengdu, Sichuan 611130; \\ ${ }^{2}$ Department of Cardiovascular Surgical, Affiliated Hospital of Weifang Medical University, \\ Weifang, Shadong 261031, P.R. China
}

Received July 5, 2021; Accepted September 16, 2021

DOI: $10.3892 /$ etm.2021.11043

\begin{abstract}
The shift in vascular function to vasoconstriction, pro-inflammatory state, oxidative stress and carbon monoxide deficiency may to endothelial dysfunction and injury, which is the key event in hypertension. Estrogen receptor modulators play a protective role in blood vessels. The present study aimed to investigate the effect of bazedoxifene, a selective estrogen receptor modulator, on human umbilical vein endothelial cells (HUVECs) and its potential underlying mechanism of action. The present study treated endothelial cells with different concentrations of bazedoxifene and determined cell viability using Cell Counting Kit- 8 to screen for the optimal working concentration of bazedoxifene. Subsequently, an angiotensin II (AngII)-induced vascular endothelial cell model was established to observe the effect of bazedoxifene on AngII-induced endothelial cells. The concentrations of nitric oxide (NO) and reactive oxygen species (ROS) were detected using NO and ROS kits, respectively. The protein expression of sirtuin 1 (SIRT1), oxidative stress-related proteins and apoptosis-related proteins was detected using western blotting, and apoptosis was detected using a TUNEL assay. The results demonstrated that bazedoxifene promoted AngII-induced HUVEC viability, reduced the expression of stress-related proteins and inhibited apoptosis. Furthermore, bazedoxifene activated SIRT1 to promote the proliferation and inhibit the oxidative stress and apoptosis of AngII-induced HUVECs. These findings suggested that bazedoxifene could effectively promote AngII-induced HUVEC proliferation and inhibit cell apoptosis and oxidative stress. In addition, bazedoxifene protected HUVEC dysfunction induced by AngII by targeting the activation of SIRT1. In summary, bazedoxifene could
\end{abstract}

Correspondence to: Dr Baotang Liu, Department of Cardiovascular Surgical, Affiliated Hospital of Weifang Medical University, 2428 YuHe Road, Weifang, Shadong 261031, P.R. China E-mail: baobaoliu211@163.com

Key words: bazedoxifene, angiotensin II-induced HUVEC, hypertension model, sirtuin 1 improve the protective role against hypertension induced by AngII.

\section{Introduction}

The complete mechanism of hypertension is complex and remains unclear. Clinical treatments [such as diet modification, increased exercise and a selection of appropriate blood pressure lowering medications (diuretics combined with angiotensin receptor antagonists or angiotensinase I inhibitors)] can effectively control the increase in high blood pressure in patients with hypertension; however, complications of hypertension can still occur, including stroke, heart failure and kidney damage (1-3). Emerging evidence has indicated that increased oxidative stress is involved in the pathogenesis of hypertension, which results in an abundance of reactive oxygen species (ROS) (4). During this process, nitric oxide (NO), synthesized by one of the NO synthase isoforms, inducible NO synthase, is a marker of oxidative stress (5). As a key enzyme in NO release, endothelial NO synthase (eNOS) also exerts a notable role in mediating oxidative stress (6). The development of hypertension is influenced by the interaction of several components, the most important being the abnormal renin-angiotensin system (RAS) (7). Angiotensin II (AngII), an important component of the RAS, can promote vasoconstriction, increase peripheral resistance and cause vascular dysfunction (8). Therefore, effective inhibition of AngII-induced vascular smooth muscle dysfunction could prevent and help the treatment of cardiovascular diseases such as hypertension.

Estrogen receptor modulators can play a vasoprotective role similar to that of estrogen (9). It has been reported that both tamoxifen and raloxifene can decrease the concentration of total serum cholesterol and low-density lipoprotein (10). Based on the reduction of lipids and inflammatory markers, tamoxifen is effective at reducing the burden of coronary heart disease (11). Following tamoxifen treatment, the mortality rate of myocardial infarction and ischemic heart disease is decreased (12). In a small group of women with high cardiovascular risk during menopause, raloxifene is associated with a reduced incidence of cardiovascular disease (13). In addition, tamoxifen can reduce the risk of heart disease and stroke; however, the risk of deep venous thrombosis development is similar to that with other selective estrogen 
receptor modulators, mainly increasing the risk of femoral vein thrombosis, pulmonary embolism and retinal venous thrombosis, although the relative incidence is very low (14).

Bazedoxifene, an estrogen receptor modulator, can alleviate cardiac hypertrophy induced by blood pressure overload in vivo by inhibiting interleukin (IL)- 6 cytokine family signal transducer signaling transduction and can reduce myocardial fibrosis induced by AngII in mice $(15,16)$. Furthermore, bazedoxifene can inhibit arterial aging and the development of atherosclerosis by increasing the expression level of sirtuin 1 (SIRT1) (17). Furthermore, SIRT1 activation can be used to treat hypertension by enhancing AMP-activated protein kinase activity (18). Therefore, the present study explored whether bazedoxifene may have the ability to attenuate AngII-induced vascular endothelial cell dysfunction by targeting the activation of SIRT1.

\section{Materials and methods}

Cell culture and treatment. Human umbilical vein endothelial cells (HUVECs) were purchased from the American Type Culture Collection (cat. no. PCS-100-010). The cells were cultured in DMEM (HyClone; Cytiva) supplemented with 10\% FBS (Gibco; Thermo Fisher Scientific, Inc.) and placed at $37^{\circ} \mathrm{C}$ in a humidified incubator with $5 \% \mathrm{CO}_{2}$.

The experiments were performed $24 \mathrm{~h}$ after induction of cells with $1 \mu \mathrm{M}$ AngII (19). Cells in the treatment group were treated with bazedoxifene $(2,4,6,8$ and $10 \mu \mathrm{M})$ for $1 \mathrm{~h}$ prior to AngII induction for $2 \mathrm{~h}$ (20). To block SIRT1, cells were incubated with $1 \mu \mathrm{M}$ of the inhibitor EX527 for $48 \mathrm{~h}$ prior to bazedoxifene treatment (21). All drugs were purchased from Sigma-Aldrich, Merck KGaA.

Cell transfection. SIRT1 small interfering (si)RNA (si-SIRT1) and siRNA negative control (si-NC) were synthesized by Shanghai GenePharma Co., Ltd. Cells were seeded into 6 -well plates at a density of $3 \times 10^{5}$ cells/well and cultured for $24 \mathrm{~h}$ at $37^{\circ} \mathrm{C}$. In strict accordance with the manufacturer's instructions, the siRNAs $(100 \mathrm{nM})$ were transfected into the cells using Lipofectamine ${ }^{\circledR} 2000$ (Invitrogen; Thermo Fisher Scientific, Inc.). The transfection efficiency was detected using reverse transcription quantitative (RT-q)PCR $48 \mathrm{~h}$ following transfection. The sequences of the siRNAs were as follows: si-SIRT1-1, 5'-GCTAAGAATTTCAGGATTA-3'; si-SIRT1-2, 5'-ACTTTGCTGTAACCCTGTA-3'; si-NC, 5'-ACUUUC AUAAGUCUUCGUGGG-3'.

Cell Counting Kit (CCK)-8 assay. Trypsin (Beyotime Institute of Biotechnology) was used to digest HUVECs and prepare a single cell suspension. Subsequently, cells were seeded into 96 -well plates at the density of $2 \times 10^{3}$ cells/well for $24 \mathrm{~h}$. A total of $10 \mu \mathrm{l} \mathrm{CCK-8} \mathrm{solution} \mathrm{(Dojindo} \mathrm{Molecular}$ Technologies, Inc., Japan) was then added to the each well of the 96-well plates. Cells were further incubated for $1 \mathrm{~h}$ and the absorbance at $450 \mathrm{~nm}$ was detected using a spectrophotometer (Thermo Fisher Scientific, Inc.).

NO and ROS determination. NO and ROS production were evaluated using NO assay kit (cat. no. BC1475) and ROS (CA1410-100T) assay kit (Beijing Solarbio Science \&
Technology). Briefly, for NO determination, cells were treated with the extract from the kit, sonicated in an ice bath for $3 \mathrm{~min}$ and centrifuged at $4^{\circ} \mathrm{C}$ for $15 \mathrm{~min}$ at $12,000 \mathrm{x} \mathrm{g}$. The absorbance of the centrifuged supernatant at $550 \mathrm{~nm}$ was measured using a spectrophotometer to determine NO production. For ROS determination, DCFH-DA was diluted 1:1,000 with serum-free medium (HyClone; Cytiva) to a final concentration of $10 \mu \mathrm{mol} / 1$. After removing the cell culture medium, enough diluted DCFH-DA was added to cover the volume of cells. They were then incubated for $20 \mathrm{~min}$ at $37^{\circ} \mathrm{C}$ in a cell incubator. DCFH-DA was removed, and the cells were washed with serum-free medium. Finally, the fluorescence intensity at $525 \mathrm{~nm}$ emission wavelength (the absorbance) was measured.

TUNEL assay. Apoptosis was detected using a TUNEL Apoptosis Assay kit (Beyotime Institute of Biotechnology). Briefly, HUVECs were washed with PBS, fixed with $4 \%$ paraformaldehyde for $20 \mathrm{~min}$ and treated with $0.1 \%$ Triton $\mathrm{X}-100$ for $10 \mathrm{~min}$, all at room temperature. Cells were incubated with TUNEL assay solution (TdT enzyme: Fluorescent labeling solution=1:9) at $37^{\circ} \mathrm{C}$ for $1 \mathrm{~h}$. Cell nucleus were stained with $10 \mu \mathrm{g} / \mathrm{ml} \mathrm{DAPI}$ for $5 \mathrm{~min}$ at $37^{\circ} \mathrm{C}$. Fluorescence expression of the apoptotic cells was observed under an EVO fluorescence microscope (Advanced Microscopy Group), and five fields of view were randomly observed in each group. The green fluorescence was considered to stain apoptotic cells and the blue stained nuclei, and the cells were counted using ImageJ (V1.8.0.112; National Institutes of Health). The apoptotic rate was calculated as follows: Apoptosis rate $=$ (average number of apoptotic cells/average number of total cells) x100\%.

$R T-q P C R$. Total RNA was extracted from cells using TRIzol ${ }^{\circledR}$ (Invitrogen; Thermo Fisher Scientific, Inc.). A reverse transcription kit (Takara Bio, Inc.) was subsequently used to reverse transcribe the RNA into cDNA according to the manufacturer's instructions. RT-qPCR reactions were performed using the ABI 7500 system (Applied Biosystems; Thermo Fisher Scientific, Inc.). The thermocycling conditions were as follows: $95^{\circ} \mathrm{C}$ for $30 \mathrm{sec}$, followed by 40 cycles of $95^{\circ} \mathrm{C}$ for $10 \mathrm{sec}$ and $60^{\circ} \mathrm{C}$ for $30 \mathrm{sec}$. The relative expression levels were normalized to endogenous control and were expressed as $2^{-\Delta \Delta \mathrm{Cq}}(22)$. The sequences of the primers were as follows: SIRT1 forward, 5'-TATGGCTGACTTCGCTTTGG-3', reverse, 5'-TCGGGG CACTGATTTCTGTA-3'; and GAPDH forward, 5'-GGAGCG AGATCCCTCCAAAAT-3' and reverse, 5'-GGCTGTTGT CATACTTCTCATGG-3'.

Western blotting. Cells were lysed with RIPA lysate (Beyotime Institute of Biotechnology) containing $1 \%$ protease inhibitor and $1 \%$ phosphatase inhibitor (both from Solarbio), centrifuged at $4^{\circ} \mathrm{C}$ for 5 min with 3,220 $\mathrm{x} \mathrm{g}$ and BCA (Beyotime Institute of Biotechnology) was used to quantify the protein concentration in the supernatant. Proteins (40 $\mu \mathrm{g} /$ lane) were separated by $10 \%$ SDS-PAGE (Beyotime Institute of Biotechnology) and transferred onto PVDF membranes (MilliporeSigma). Subsequently, membranes were blocked with 5\% skimmed milk for $2 \mathrm{~h}$ at room temperature and incubated with primary antibodies against phosphorylated-eNOS (p-eNOS; 1:300; cat. no. ab215717; Abcam), eNOS (1:400; cat. no. ab252439; 
Abcam), p47 phox (1:300; cat. no. ab181090; Abcam), Bcl-2 (1:400; cat. no. ab182858; Abcam), Bax (1:400; cat. no. ab32503; Abcam), cleaved caspase-3 (1:500; cat. no. ab32042; Abcam), caspase 3, (1:500; cat. no. ab32351; Abcam), SIRT1 (1:400; cat. no. ab189494; Abcam), $\beta$-actin (1:1,000; cat. no. ab8227; Abcam) and GAPDH (1:1,000; cat. no. ab181602; Abcam) overnight at $4^{\circ} \mathrm{C}$. Subsequently, the membranes were washed with PBS-10\% Tween-20 three times and were incubated with the goat anti-rabbit IgG H\&L (HRP; 1:1,000; cat. no. ab7090; Abcam) for $2 \mathrm{~h}$ at room temperature. Pierce Western Blotting Substrate (Thermo Fisher Scientific, Inc.) was used to detect the signal on the membrane. The data were analyzed via densitometry using ImageJ software and normalized to expression of the internal controls $\beta$-actin or GAPDH.

Statistical analysis. Each experiment was repeated independently at least three times. The data are presented as the means \pm standard deviation. Statistical analyses were performed using SPSS v19.0 software (IBM Corp.). One-way ANOVA followed by Tukey's post hoc test was used for statistical analyses. $\mathrm{P}<0.05$ was considered to indicate a statistically significant difference.

\section{Results}

Bazedoxifene promotes the proliferation of HUVECs induced by AngII. The effect of bazedoxifene on normal endothelial cells was determined, as shown in Fig. 1A. The results from CCK-8 assay demonstrated that low concentrations of bazedoxifene had little effect on cell viability. However, when the concentration of bazedoxifene was $>6 \mu \mathrm{M}$, cell viability was significantly decreased, demonstrating a cytotoxicity of bazedoxifene. Subsequently, the concentrations of 2-6 $\mu \mathrm{M}$ bazedoxifene were selected in subsequent experiments. The cells were then pretreated with bazedoxifene for $1 \mathrm{~h}$, followed by treatment with $1 \mu \mathrm{M}$ AngII for $24 \mathrm{~h}$. The results from CCK-8 assay showed that bazedoxifene promoted the viability of HUVECs induced by AngII (Fig. 1B).

Bazedoxifene reduces AngII-induced oxidative stress in endothelial cells. The effect of bazedoxifene on AngII-induced endothelial cells was further investigated, and NO content was detected using a NO kit. As presented in Fig. 1C, bazedoxifene upregulated the decreased NO content in Ang-II-induced HUVECs in a concentration-dependent manner. Furthermore, bazedoxifene treatment decreased the ROS content in AngII-induced endothelial cells (Fig. 1D). Subsequently, the expression levels of oxidative stress-related proteins were detected using western blotting. The protein expression levels of p-eNOS and eNOS were decreased, while the protein expression levels of p47 phox were increased in AngII-induced endothelial cells. Furthermore, the protein expression levels of p-eNOS and eNOS were increased, while the protein expression level of $\mathrm{p} 47$ phox was decreased following the addition of bazedoxifene (Fig. 1E). These findings indicated that bazedoxifene may reduce AngII-induced oxidative stress in endothelial cells.

Bazedoxifene inhibits AngII-induced apoptosis of endothelial cells. To determine the effect of bazedoxifene on the apoptosis of AngII-induced HUVECs, TUNEL staining was performed. The results demonstrated that the apoptotic rate in the AngII-treated alone group was significantly higher compared with that in the control group; however, bazedoxifene treatment could decrease cell apoptosis (Fig. 2A and B). These results were further confirmed by the detection of apoptosis-related protein expression levels. As presented in Fig. 2C and D, bazedoxifene could decrease Bax and cleaved caspase-3 protein expression levels in the AngII-induced HUVECs compared with the AngII-induced HUVECs alone group; however, bazedoxifene increased the expression level of the anti-apoptosis protein $\mathrm{Bcl}-2$. These findings demonstrated that bazedoxifene may inhibited the AngII-induced apoptosis of endothelial cells.

Bazedoxifene promotes AngII-induced endothelial cell proliferation and inhibits oxidative stress by activating SIRT1. It has been reported that SIRT1 activation can be used to limit hypertension by enhancing AMP-activated protein kinase activity (23). In the present study, si-SIRT1 was transfected into HUVECs and the results from western blotting (Fig. 3A) and RT-qPCR (Fig. 3B) demonstrated that the transfection efficiency of si-SIRT1-1 was higher than of si-SIRT1-2. si-SIRT1-1 was therefore selected for use in subsequent experiments. The expression level of SIRT1 was decreased in AngII-induced endothelial cells, while the expression level of SIRT1 was increased with bazedoxifene in a concentration-dependent manner. The results showed that when the concentration of bazedoxifene was $4 \mu \mathrm{M}$, the inhibitory effect of AngII on SIRT1 was counteracted (Fig. 3C and D). Therefore, this concentration was selected for use in subsequent experiments.

The SIRT1 inhibitor, EX527, was added to the AngII-induced endothelial cells. The results from CCK-8 assay demonstrated that si-SIRT1 could partially counteract the promoting effect of bazedoxifene on the proliferation of AngII-induced endothelial cells (Fig. 3E). These results indicated that bazedoxifene may activate SIRT1 and promote AngII-induced endothelial cell proliferation.

Furthermore, as presented in Fig. 3F, bazedoxifene could increase the concentration of NO, while si-siRT1 and EX527 could partially reduce the concentration of NO. Furthermore, bazedoxifene could decrease ROS content, whereas both si-SIRT1 and EX527 partially increased the concentration of ROS (Fig. 3G). The results from western blotting further demonstrated that bazedoxifene could activate SIRT1 to inhibit AngII-induced oxidative stress in endothelial cells (Fig. 3H).

Bazedoxifene activates SIRT1 to inhibit AngII-induced apoptosis of endothelial cells. As presented in Fig. 4A and B, the cell apoptosis rate in the bazedoxifene treatment group was significantly decreased compared with that in the AngII group, and both si-SIRT1 and EX527 could partially increase the apoptosis rate. These results were further confirmed by detecting the expression levels of apoptosis-related proteins (Fig. 4C and D). Both si-SIRT1 and EX527 partially increased the protein expression of the pro-apoptotic protein Bax, cleaved caspase $3 /$ caspase 3 , while promoting the degradation of the anti-apoptotic protein $\mathrm{Bcl}-2$ in endothelial cells in comparison with the AngII $+4 \mu \mathrm{g} / \mathrm{ml}+$ si-NC goup and the AngII $+4 \mu \mathrm{g} / \mathrm{ml}$ group, respectively. These findings 

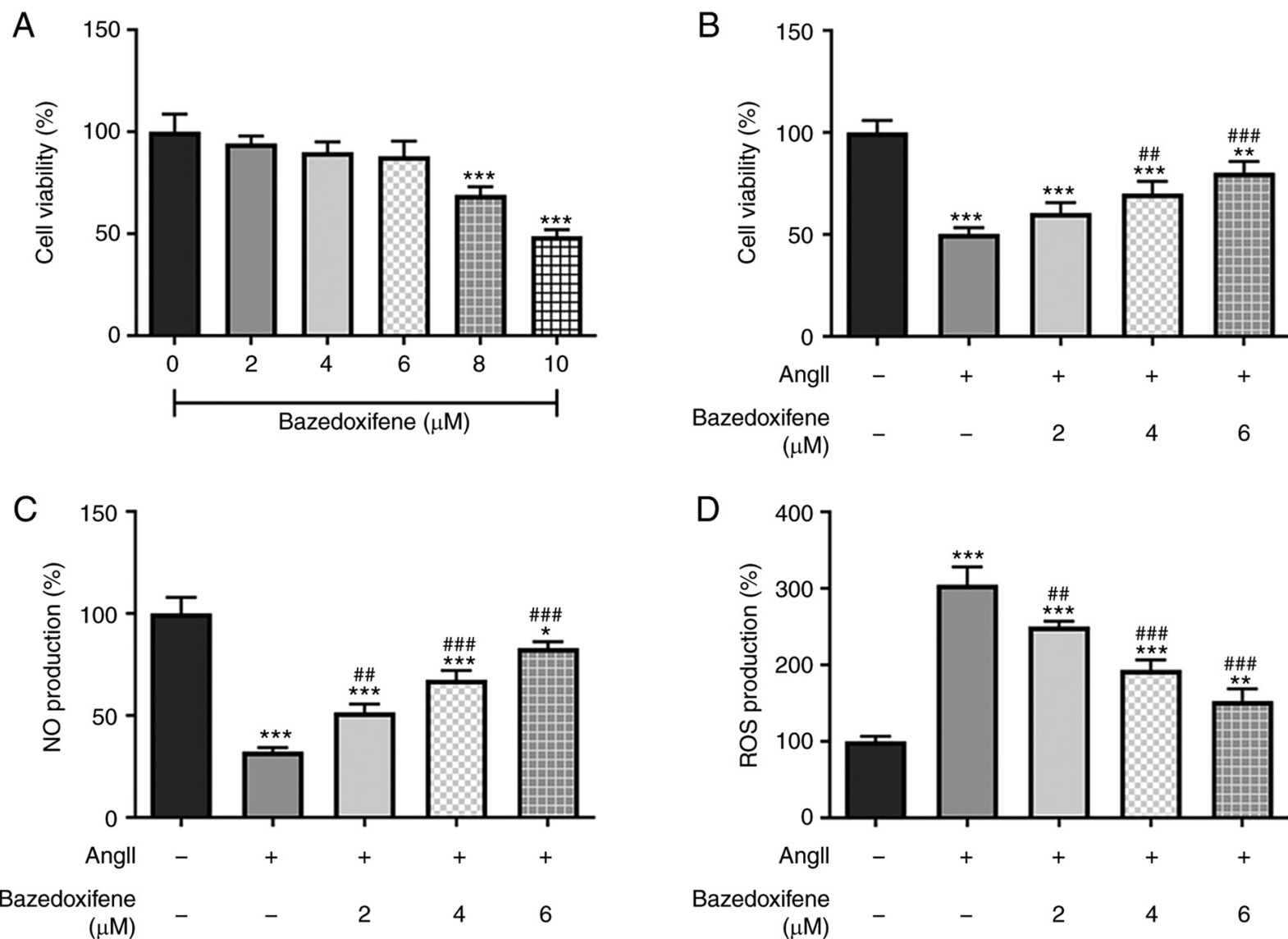

$\mathrm{E}$
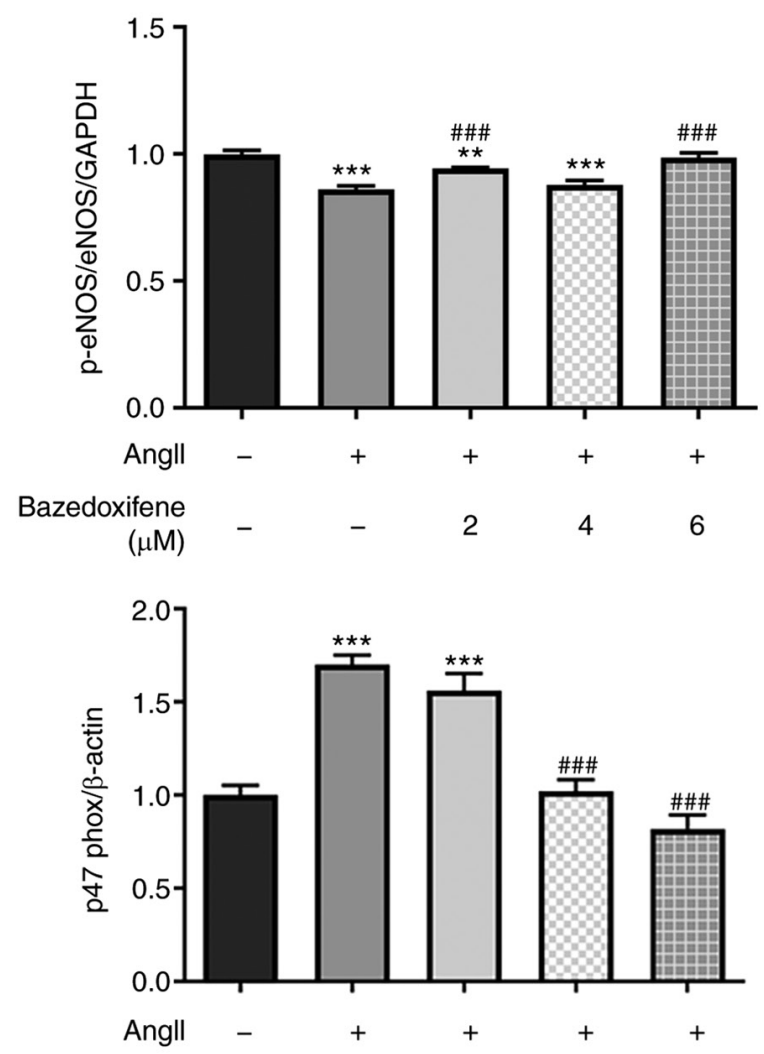

Bazedoxifene $(\mu \mathrm{M})$

Figure 1. Bazedoxifene promotes the proliferation and decreases oxidative stress in HUVEC cells induced by AngII. (A) CCK-8 assay was used to detect cell viability of HUVEC cells treated with bazedoxifene. (B) CCK-8 assay was used to detect the cell viability of HUVEC cells induced by AngII. (C) NO content was detected using a NO kit. (D) ROS content was detected using a ROS kit. (E) Expression of oxidative stress related proteins was detected by western blotting. ${ }^{*} \mathrm{P}<0.05,{ }^{* *} \mathrm{P}<0.01$ and ${ }^{* * * *} \mathrm{P}<0.001$ vs. Control; ${ }^{\# \#} \mathrm{P}<0.01$ and ${ }^{\# \# "} \mathrm{P}<0.001$ vs. AngII $+0 \mu \mathrm{g} / \mathrm{ml}$. AngII, angiotensin II; CCK-8, Cell Counting Kit-8; NO, nitric oxide; ROS, reactive oxygen species; eNOS, endothelial nitric oxide synthase; p, phosphorylated. 
A

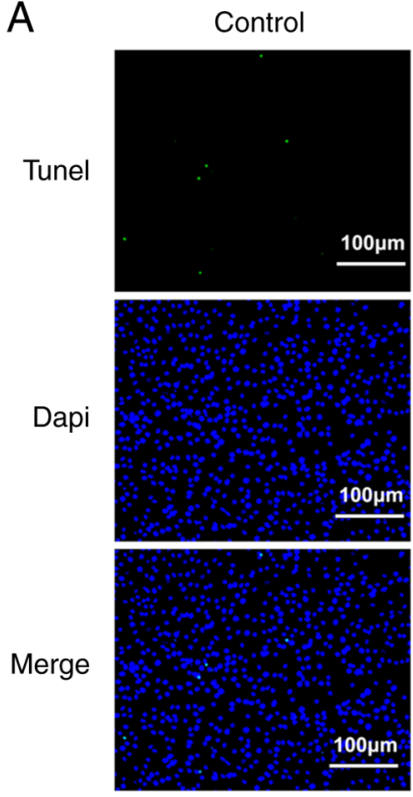

B

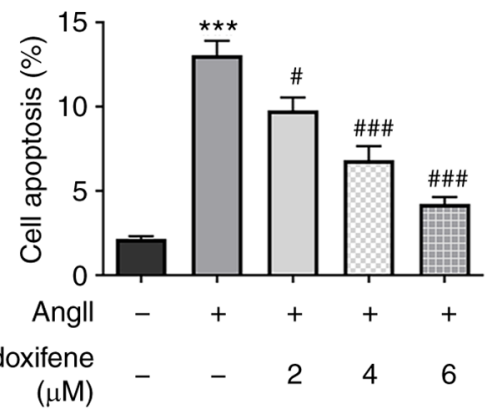

Angll $+0 \mu \mathrm{g} / \mathrm{ml}$
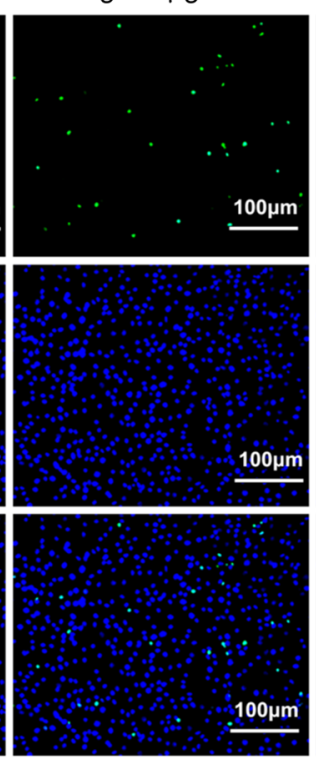

$00 \mu \mathrm{m}$
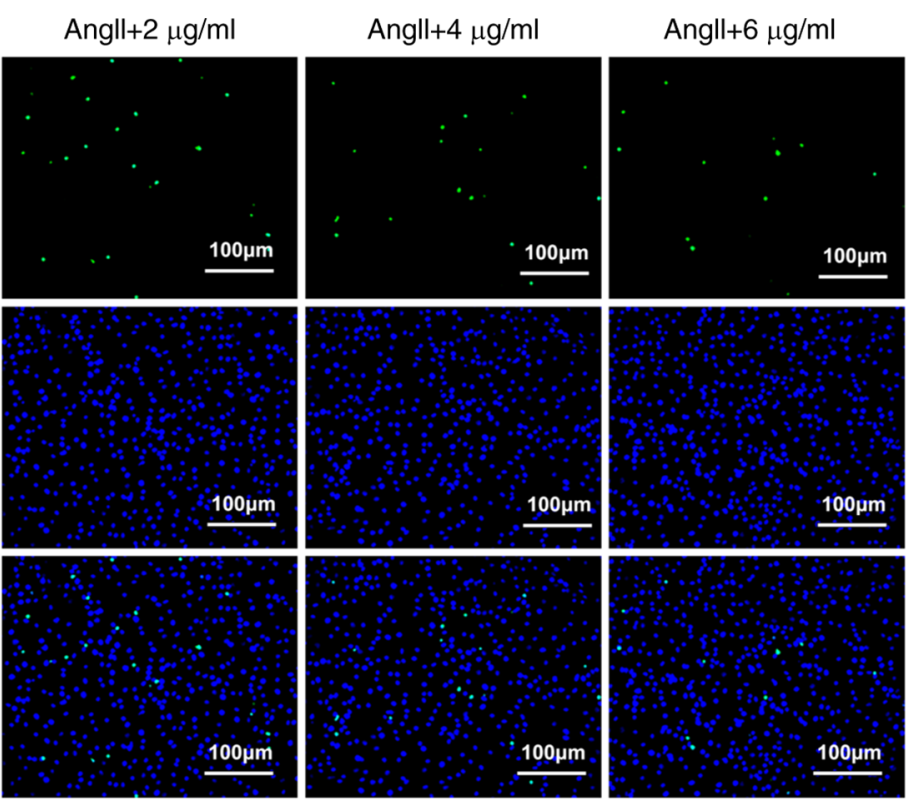

C

Cleaved caspase-3

Caspase-3

GAPDH

Angll -++++

Bazedoxifene

$(\mu \mathrm{M})$
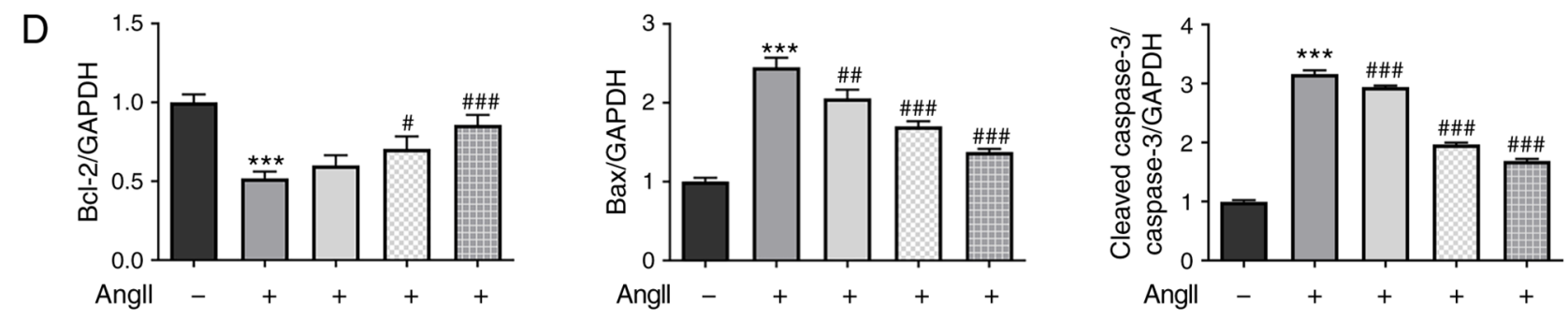

Bazedoxifene

$(\mu \mathrm{M})$

$(\mu \mathrm{M})$

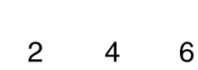

Bazedoxifene
$(\mu \mathrm{M})$

Figure 2. Bazedoxifene inhibits AngII-induced HUVEC apoptosis. (A and B) TUNEL staining was performed to detect the apoptosis of AngII-induced HUVEC cells. Magnification, scale bars, $100 \mu \mathrm{m}$. (C and D) Expression of apoptosis-related proteins was detected by western blotting. ${ }^{* * *} \mathrm{P}<0.001$ vs. Control; ${ }^{\#} \mathrm{P}<0.05,{ }^{\# \#} \mathrm{P}<0.01$ and ${ }^{\# \# \#} \mathrm{P}<0.001$ vs. AngII $+0 \mu \mathrm{g} / \mathrm{ml}$. AngI, angiotensin II.

suggested that bazedoxifene may activate SIRT1 to inhibit the AngII-induced apoptosis of endothelial cells.

\section{Discussion}

AngII is the main active peptide of RAS, and AngII produced by both classical and non-classical pathways can reduce endothelial cell function (24). Endothelial cells are the main components of the vascular system, which serve a crucial role in vascular homeostasis by secreting and releasing vasodilators (such as NO and prostaglandins) and vasoconstrictors (such as AngII and endothelin) (25-27). The shift of vascular function to vasoconstriction, proinflammatory state, oxidative stress and NO deficiency may lead to endothelial dysfunction and injury. This shift is a key event in the pathophysiological process of cardiovascular diseases, including hypertension, diabetes, atherosclerosis, arterial hypertension and pulmonary hypertension $(28,29)$. In the present study, HUVECs were treated with $1 \mu \mathrm{M}$ AngII for $24 \mathrm{~h}$ to establish an AngII-induced endothelial cell model. The results demonstrated that endothelial cell viability was decreased, while oxidative stress levels and apoptosis were increased in AngII-induced cells, which was consistent with a previous report (30).

Statins are mainly used to reduce blood lipid and cholesterol levels and treat patients with cardiovascular diseases (31). At present, the commonly used statins in 
A

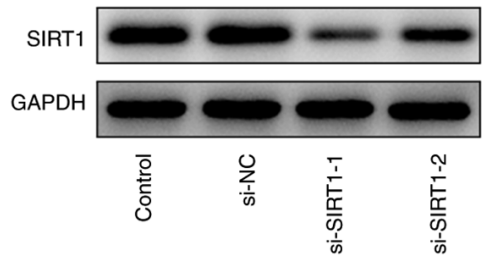

C

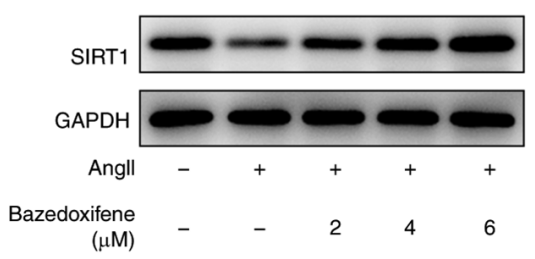

$E$

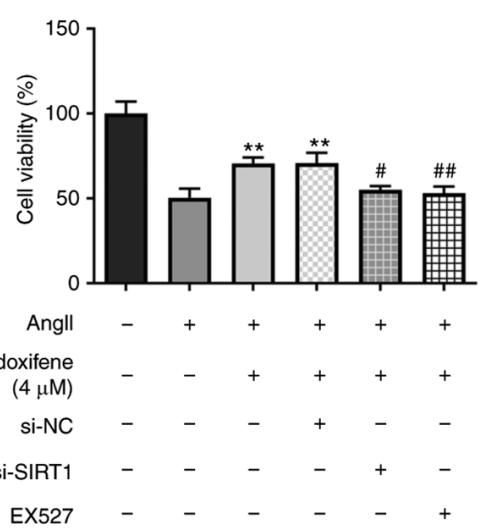

$\mathrm{H}$

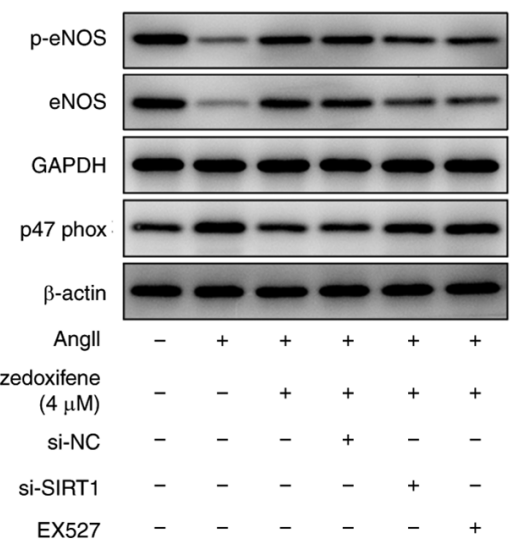

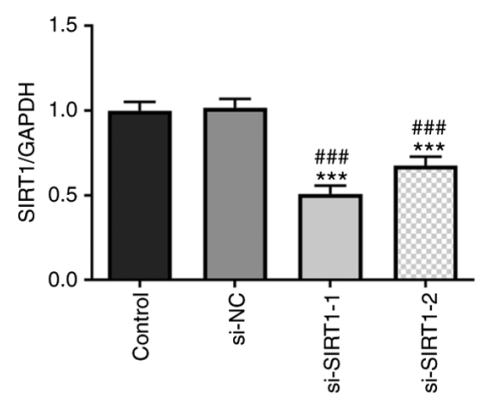

B

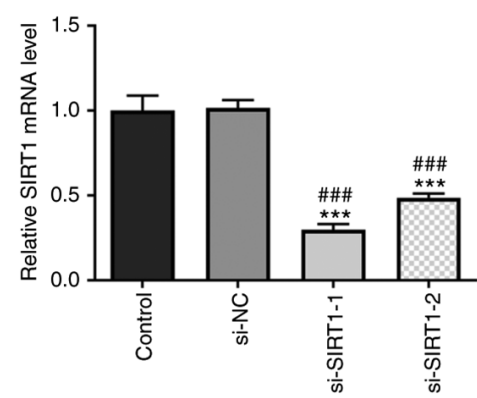

D

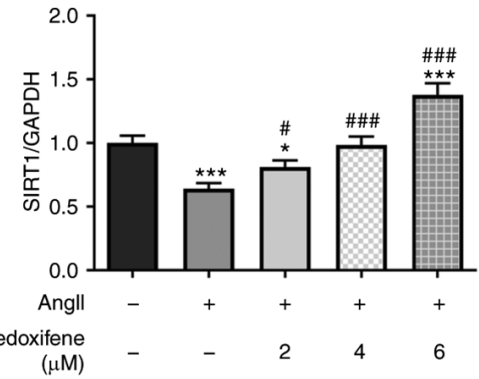

$\mathrm{F}$

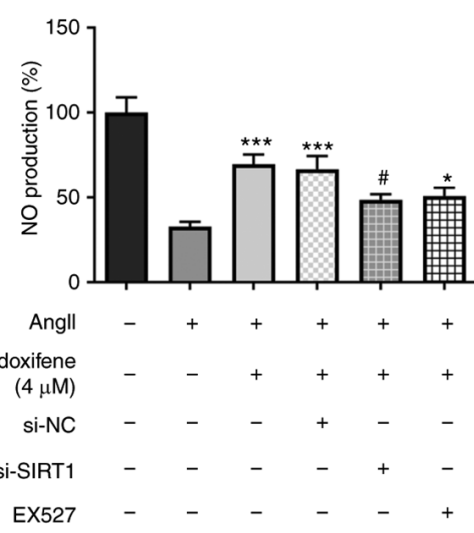

G
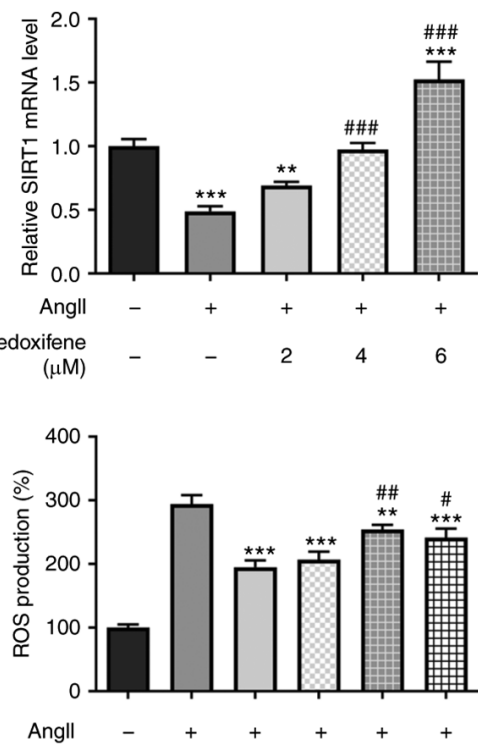

Bazedoxifene - -++++

Si-NC $-\quad-\quad+-$

si-SIRT1

EX527
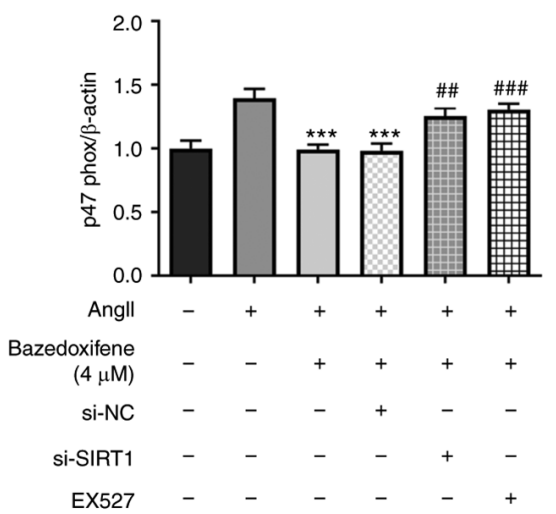

Figure 3. Bazedoxifene stimulates AngII-induced HUVEC proliferation and inhibits oxidative stress by activating SIRT1. (A and B) Cell transfection efficiency with si-SIRT1 was confirmed by (A) western blotting (A) and (B) RT-qPCR. (C and D) Expression of SIRT1 in AngII-induced endothelial cells was detected by (C) western blotting and (D) RT-qPCR. (E) Cell Counting Kit-8 assay was used to determine the cell viability. (F) NO content was detected using a NO kit. (G) ROS content was detected using a ROS kit. (H) Expression of oxidative stress related proteins was detected by western blotting. ${ }^{*} \mathrm{P}<0.05$, ${ }^{* * *} \mathrm{P}<0.01$ and ${ }^{* * * *} \mathrm{P}<0.001$ vs. Control or AngII $+0 \mu \mathrm{g} / \mathrm{ml} ;{ }^{\#} \mathrm{P}<0.05,{ }^{\# \#} \mathrm{P}<0.01$ and ${ }^{\# \# "} \mathrm{P}<0.001$ vs. si-NC or AngII $+4 \mu \mathrm{g} / \mathrm{ml}+$ si-NC. RT-qPCR, reverse transcription quantitative PCR; ANGII, angiotensin II; SIRT1, sirtuin 1; NO, nitric oxide; ROS, reactive oxygen species; eNOS, endothelial nitric oxide synthase; p, phosphorylated; si, small interfering; NC, negative control.

clinical practice include atorvastatin, fluvastatin, lovastatin, simvastatin and pravastatin. Recent studies have reported that statins exert anti-inflammatory and anti-angiogenesis effects and can inhibit endothelial cell migration $(32,33)$. The results from the present study demonstrated that bazedoxifene could promote AngII-induced HUVEC proliferation and inhibit cell apoptosis, indicating that bazedoxifene could enhance the viability of AngII-induced HUVEC.

When the endothelium suffers damage and dysfunction (such as ischemia and oxygen deprivation reperfusion), the 
A

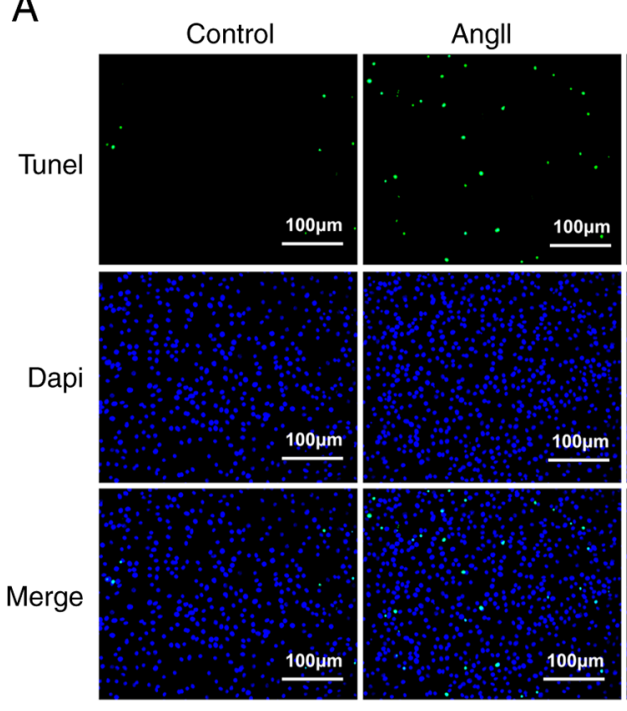

B
Angll+4 $\mu \mathrm{g} / \mathrm{ml}$

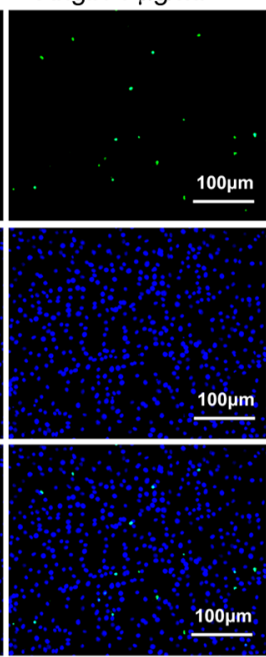

$100 \mu \mathrm{m}$
Angll+4 $\mu \mathrm{g} / \mathrm{ml}+$ si-NC

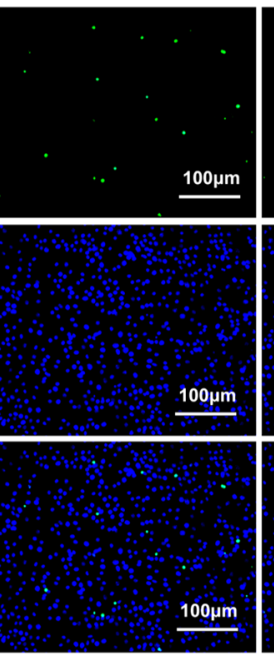

C

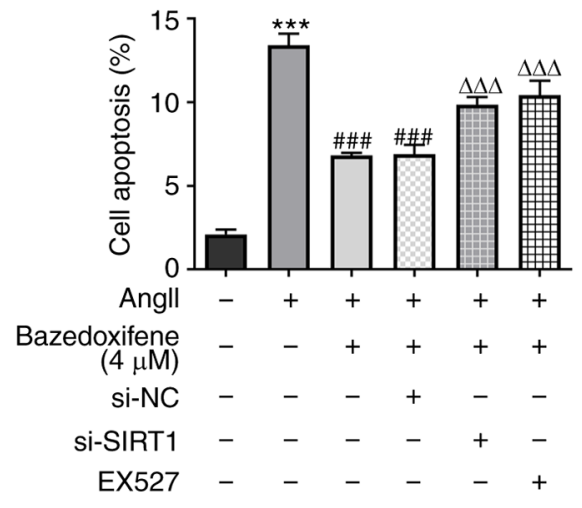

D

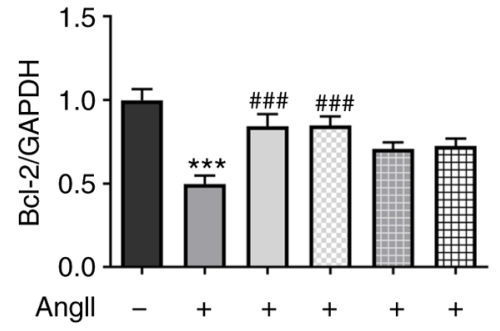

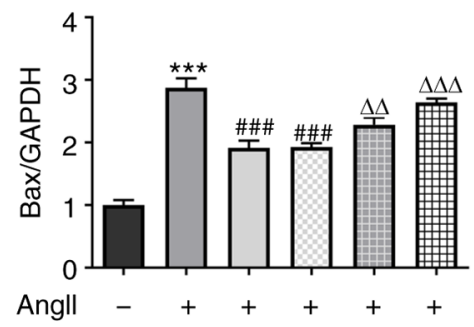

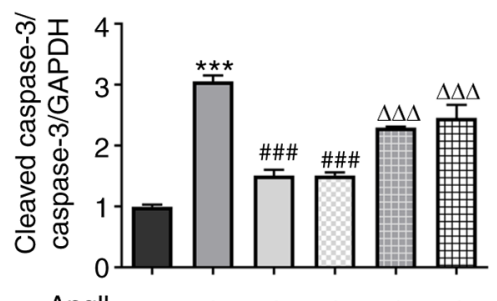

Bazedoxifene $-\quad+\quad+\quad+\quad+$ Bazedoxifene si-NC

Si-SIRT1 
of atherosclerosis and vascular senescence (37). In addition, SIRT1 can reduce systolic blood pressure elevation and inhibit AngII-induced vascular remodeling in mice (38). These results might indicate whether bazedoxifene mechanism of action could be mediated by targeted agonism of SIRT1. Therefore, to further investigate bazedoxifene underlying mechanism, the present study determined the effect of bazedoxifene on AngII-induced cells using STIR1 inhibitor and si-SIRT1. The results demonstrated that both EX527 and si-SIRT1 could counteract the apoptosis-inhibiting effect and anti-oxidative stress effect observed with bazedoxifene. Subsequently, our study suggested that bazedoxifene may be able to counteract AngII-induced endothelial cell apoptosis, increased oxidative stress and NO release dysfunction by agonizing SIRT1. However, the present study only focused on the protective effect of bazedoxifene on AngII-induced cells by inhibiting or downregulating STIR1. Future investigation will involve animal experiments to confirm these results, refine our understanding and provide an experimental basis for the application of bazedoxifene in the treatment of AngII-induced diseases.

In summary, the present study demonstrated that bazedoxifene may promote AngII-induced HUVEC proliferation and inhibit cell apoptosis and oxidative stress by activating SIRT1, thereby playing a protective role. These findings could provide an important basis for the clinical treatment of AngII-induced hypertension with estrogen.

\section{Acknowledgements}

Not applicable.

\section{Funding}

No funding was received.

\section{Availability of data and materials}

The datasets used and/or analyzed during the current study are available from the corresponding author on reasonable request.

\section{Authors' contributions}

QY and BL designed the study and wrote the manuscript. QY and BL confirm the authenticity of all the raw data. QY, JZ and BL performed experiments and participated in data collection and analysis. All authors have read and approved the final manuscript.

\section{Ethics approval and consent to participate}

Not applicable.

\section{Patient consent for publication}

Not applicable.

\section{Competing interests}

The authors declare that they have no competing interests.

\section{References}

1. Brumback LC, Andrews LIB, Jacobs DR Jr, Duprez DA, Shah SJ, Dougherty CM, Denenberg JO and Allison MA: The association between indices of blood pressure waveforms (PTC1 and PTC2) and incident heart failure. J Hypertens 39: 661-666, 2021.

2. Harrison N, Pang P, Collins S and Levy P: Blood pressure reduction in hypertensive acute heart failure. Curr Hypertens Rep 23: $11,2021$.

3. Hering D, Esler MD, Krum H, Mahfoud F, Böhm M, Sobotka PA and Schlaich MP: Recent advances in the treatment of hypertension. Expert Rev Cardiovase Ther 9: 729-744, 2011.

4. Rodrigo R, González J and Paoletto F: The role of oxidative stress in the pathophysiology of hypertension. Hypertens Res 34: 431-440, 2011.

5. Pierini D and Bryan NS: Nitric oxide availability as a marker of oxidative stress. Methods Mol Biol 1208: 63-71, 2015.

6. Wang ZM, Zhang JX, Li B, Gao X, Liu Y, Mao W and Chen SL: Resveratrol ameliorates low shear stress-induced oxidative stress by suppressing ERK/eNOS-Thr495 in endothelial cells. Mol Med Rep 10: 1964-1972, 2014.

7. Li XC, Zhang J and Zhuo JL: The vasoprotective axes of the renin-angiotensin system: Physiological relevance and therapeutic implications in cardiovascular, hypertensive and kidney diseases. Pharmacol Res 125: 21-38, 2017.

8. Fyhrquist $\mathrm{F}$ and Saijonmaa O: Renin-angiotensin system revisited. J Intern Med 264: 224-236, 2008.

9. Lee HS, Park HK, Kim KH, Ko JH, Kim YJ, Yi KH and Hwang JS: Estrogen receptor $\alpha$ gene analysis in girls with central precocious puberty. J Pediatr Endocrinol Metab 26: 645-649, 2013.

10. Demissie S, Cupples LA, Shearman AM, Gruenthal KM, Peter I, Schmid CH, Karas RH, Housman DE, Mendelsohn ME and Ordovas JM: Estrogen receptor-alpha variants are associated with lipoprotein size distribution and particle levels in women: The Framingham heart study. Atherosclerosis 185: 210-218, 2006.

11. Chlebowski RT, Anderson GL, Geller M and Col N: Coronary heart disease and stroke with aromatase inhibitor, tamoxifen, and menopausal hormone therapy use. Clin Breast Cancer 6 (Suppl 2): S58-S64, 2006.

12. Nordenskjold B, Rosell J, Rutqvist LE, Malmström PO, Bergh J, Bengtsson NO, Hatschek T, Wallgren A and Carstensen J: Coronary heart disease mortality after 5 years of adjuvant tamoxifen therapy: Results from a randomized trial. J Natl Cancer Inst 97: 1609-1610, 2005.

13. Sharma D, Sharma U, Bhatnagar VB and Singh VS: A study of the effect of tamoxifen on serum lipoprotein profiles in premenopausal and postmenopausal women with breast carcinoma and associated risk of cardiovascular disease. Indian J Med Sci 55: 359-365, 2001.

14. McLean BA, Zhabyeyev P, Patel VB, Basu R, Parajuli N, DesAulniers J, Murray AG, Kassiri Z, Vanhaesebroeck B and Oudit GY: PI3Kalpha is essential for the recovery from Cre/tamoxifen cardiotoxicity and in myocardial insulin signalling but is not required for normal myocardial contractility in the adult heart. Cardiovasc Res 105: 292-303, 2015.

15. Shi W, Ma H, Liu T, Yan D, Luo P, Zhai M, Tao J, Huo S, Guo J, Li C, et al: Inhibition of Interleukin-6/glycoprotein 130 signalling by Bazedoxifene ameliorates cardiac remodelling in pressure overload mice. J Cell Mol Med 24: 4748-4761, 2020.

16. McKeand W, Ermer J and Korth-Bradley J: Assessment of the effects of age and renal function on pharmacokinetics of bazedoxifene in postmenopausal women. Clin Pharmacol Drug Dev 7: 920-926, 2018.

17. Clarkson TB, Ethun KF, Pajewski NM, Golden D, Floyd E and Appt SE: Effects of bazedoxifene, conjugated equine estrogens, and a tissue-selective estrogen complex containing both bazedoxifene and conjugated equine estrogens on cerebral artery atherosclerosis in postmenopausal monkeys. Menopause 21: 8-14, 2014.

18. Martinez-Arroyo O, Ortega A, Galera M, Solaz E, Martinez-Hervas S, Redon J and Cortes R: Decreased urinary levels of SIRT1 as non-invasive biomarker of early renal damage in hypertension. Int J Mol Sci 21: 6390, 2020.

19. Yang R, Fang W, Liang J, Lin C, Wu S, Yan S, Hu C and Ke X: Apelin/APJ axis improves angiotensin II-induced endothelial cell senescence through AMPK/SIRT1 signaling pathway. Arch Med Sci 14: 725-734, 2018. 
20. Ishibashi Y, Matsui T, Ueda S, Fukami K, Okuda S, Ohta H and Yamagishi S: Bazedoxifene blocks AGEs-RAGE-induced superoxide generation and MCP-1 level in endothelial cells. Climacteric 18: 426-430, 2015.

21. Wang XY, Li XY, Wu CH, Hao Y, Fu PH, Mei HX, Chen F, Gong YQ, Jin SW and $\mathrm{Li} \mathrm{H}$ : Protectin conjugates in tissue regeneration 1 restores lipopolysaccharide-induced pulmonary endothelial glycocalyx loss via ALX/SIRT1/NF-kappa B axis. Respir Res 22: 193, 2021.

22. Livak KJ and Schmittgen TD: Analysis of relative gene expression data using real-time quantitative PCR and the 2(-Delta Delta C(T)) method. Methods 25: 402-408, 2002.

23. Gao D, Zuo Z, Tian J, Ali Q, Lin Y, Lei H and Sun Z: Activation of SIRT1 attenuates klotho deficiency-induced arterial stiffness and hypertension by enhancing AMP-activated protein kinase activity. Hypertension 68: 1191-1199, 2016.

24. Ding Y, Chen J, Cui G, Wei Y, Lu C, Wang L and Diao H: Pathophysiological role of osteopontin and angiotensin II in atherosclerosis. Biochem Biophys Res Commun 471: 5-9, 2016.

25. Wei F, Liu S, Luo L, Gu N, Zeng Y, Chen X, Xu S and Zhang D: Anti-inflammatory mechanism of ulinastatin: Inhibiting the hyperpermeability of vascular endothelial cells induced by TNF- $\alpha$ via the RhoA/ROCK signal pathway. Int Immunopharmacol 46 : 220-227, 2017

26. Yamawaki H, Kameshima S, Usui T, Okada M and Hara Y: A novel adipocytokine, chemerin exerts anti-inflammatory roles in human vascular endothelial cells. Biochem Biophys Res Commun 423: 152-157, 2012

27. Fan X, Wang E, He J, Zhang L, Zeng X, Gui Y, Sun Q, Song Y and Yuan H: Ligustrazine protects homocysteine-induced apoptosis in human umbilical vein endothelial cells by modulating mitochondrial dysfunction. J Cardiovasc Transl Res 12: 591-599, 2019.

28. Chan P, Chen YC, Lin LJ, Cheng TH, Anzai K, Chen YH, Liu ZM, Lin JG and Hong HJ: Tanshinone IIA attenuates $\mathrm{H}(2) \mathrm{O}(2)$-induced injury in human umbilical vein endothelial cells. Am J Chin Med 40: 1307-1319, 2012.

29. Bik E, Mielniczek N, Jarosz M, Denbigh J, Budzynska R, Baranska M and Majzner K: Tunicamycin induced endoplasmic reticulum changes in endothelial cells investigated in vitro by confocal Raman imaging. Analyst 144: 6561-6569, 2019.
30. Wassmann S, Laufs U, Stamenkovic D, Linz W, Stasch JP, Ahlbory K, Rösen R, Böhm M and Nickenig G: Raloxifene improves endothelial dysfunction in hypertension by reduced oxidative stress and enhanced nitric oxide production. Circulation 105: 2083-2091, 2002.

31. Koushki K, Shahbaz SK, Mashayekhi K, Sadeghi M, Zayeri ZD, Taba MY, Banach M, Al-Rasadi K, Johnston TP and Sahebkar A: Anti-inflammatory action of statins in cardiovascular disease: The role of inflammasome and toll-like receptor pathways. Clin Rev Allergy Immunol 60: 175-199, 2021.

32. Mostafa TM, Hegazy SK, Elshebini EM, Saif DS and Elabd AH: A comparative study on the anti-inflammatory effect of angiotensin-receptor blockers \& statins on rheumatoid arthritis disease activity. Indian J Med Res 152: 393-400, 2020

33. Peppas S, Piovani D, Peyrin-Biroulet L, Danese S and Bonovas S: Statins and inflammatory bowel disease: Where do we stand? Eur J Intern Med 75: 10-14, 2020.

34. Orellana-Urzúa S, Rojas I, Líbano L and Rodrigo R Pathophysiology of ischemic stroke: Role of oxidative stress. Curr Pharm Des 26: 4246-4260, 2020

35. Vanhoutte PM: Nitric oxide: From good to bad. Ann Vasc Dis 11: 41-51, 2018.

36. $\mathrm{Li} \mathrm{H}$ and Forstermann U: Nitric oxide in the pathogenesis of vascular disease. J Pathol 190: 244-254, 2000.

37. Sasaki Y, Ikeda Y, Miyauchi T, Uchikado Y, Akasaki Y and Ohishi M: Estrogen-SIRT1 axis plays a pivotal role in protecting arteries against menopause-induced senescence and atherosclerosis. J Atheroscler Thromb 27: 47-59, 2020.

38. Li L, Gao P, Zhang H, Chen H, Zheng W, Lv X, Xu T, Wei Y, Liu D and Liang C: SIRT1 inhibits angiotensin II-induced vascular smooth muscle cell hypertrophy. Acta Biochim Biophys Sin (Shanghai) 43: 103-109, 2011.

(i) $\Theta$ This work is licensed under a Creative Commons Attribution-NonCommercial-NoDerivatives 4.0 International (CC BY-NC-ND 4.0) License. 